Portland State University

PDXScholar

$12-2018$

\title{
Dispersion Analysis of HDG Methods
}

Jay Gopalakrishnan

Portland State University, gjay@pdx.edu

Manuel Solano

Universidad de Concepción

Felipe Vargas

Universidad de Concepción

Follow this and additional works at: https://pdxscholar.library.pdx.edu/mth_fac

Part of the Mathematics Commons

Let us know how access to this document benefits you.

\section{Citation Details}

Gopalakrishnan, J., Solano, M., \& Vargas, F. (2018). Dispersion analysis of HDG methods. Journal of Scientific Computing, 77(3), 1703. Retrieved from http://stats.lib.pdx.edu/ proxy.php?url=http://search.ebscohost.com/ login.aspx?direct=true\&db=msn\&AN=MR3874791\&site=ehost-live

This Post-Print is brought to you for free and open access. It has been accepted for inclusion in Mathematics and Statistics Faculty Publications and Presentations by an authorized administrator of PDXScholar. Please contact us if we can make this document more accessible: pdxscholar@pdx.edu. 


\title{
Dispersion analysis of HDG methods
}

\author{
Jay Gopalakrishnan • Manuel Solano • \\ Felipe Vargas
}

Received: date / Accepted: date

\begin{abstract}
This work presents a dispersion analysis of the Hybrid Discontinuous Galerkin (HDG) method. Considering the Helmholtz system, we quantify the discrepancies between the exact and discrete wavenumbers. In particular, we obtain an analytic expansion for the wavenumber error for the lowest order Single Face HDG (SFH) method. The expansion shows that the SFH method exhibits convergence rates of the wavenumber errors comparable to that of the mixed hybrid Raviart-Thomas method. In addition, we observe the same behavior for the higher order cases in numerical experiments.
\end{abstract}

Keywords dispersion analysis • hybridizable discontinuous Galerkin • Helmholtz equation

J. Gopalakrishnan

F. Maseeh Department of Mathematics \& Statistics Portland State University, PO Box 751, Portland, OR 97207-0751, USA.

E-mail: gjay@pdx.edu

M. Solano

Departamento de Ingeniería Matemática and Centro de Investigación en Ingeniería Matemática ( $\left.\mathrm{CI}^{2} \mathrm{MA}\right)$, Universidad de Concepción, Concepción, Chile.

E-mail: msolano@ing-mat.udec.cl

F. Vargas

Departamento de Ingeniería Matemática and Centro de Investigación en Ingeniería Matemática ( $\left.\mathrm{CI}^{2} \mathrm{MA}\right)$, Universidad de Concepción, Concepción, Chile.

E-mail: fvargas@ci2ma.udec.cl 


\section{Introduction}

In many physical and engineering applications involving wave propagation, the mathematical model is governed by the Helmholtz system

$$
\begin{aligned}
i k \mathbf{u}+\nabla \phi=\mathbf{0} & \text { in } \Omega, \\
i k \phi+\nabla \cdot \mathbf{u}=f & \text { in } \Omega, \\
\phi=0 & \text { on } \partial \Omega,
\end{aligned}
$$

where $0 \neq k \in \mathbb{C}$ is the wavenumber, $\Omega \subset \mathbb{R}^{2}, \mathbf{u}: \Omega \rightarrow \mathbb{R}^{2}$ is a vector unknown, $\phi: \Omega \rightarrow \mathbb{R}$ is a scalar unknown, and $f \in L^{2}(\Omega)$ is a given source term. For example, in acoustics, $\mathbf{u}$ represents linearized velocity and $\phi$ represents pressure. When solving the Helmholtz system using a numerical method, the so-called "pollution effect" [4] manifests itself in discrepancies between the method's discrete wavenumber and the exact $k$. Dispersion analyses have long been used to determine these discrepancies for standard methods (see $[1,9]$ and the bibliography therein).

In a dispersion analysis, one attempts to propagate a wave of exact wavenumber $k$ using a numerical method on an infinite uniform mesh of grid size $h>0$. The equations of the method then show that the numerical solution can be viewed as a wave of a possibly different wavenumber $k^{h}$. Thus, the difference between $k$ and $k^{h}$ quantifies the wavenumber error of the method. It is traditional to study these differences in the following three forms: "dispersive error" $\operatorname{Re}\left(k h-k^{h} k\right)$, "dissipative error" $\operatorname{Im}\left(k h-k^{h} k\right)$, and the "total error" $\left|k h-k^{h} h\right|$. The behaviour of these quantities as $k h$ goes to 0 gives us valuable insights into the method.

Many previous works have focused on dispersion analyses of various numerical methods [1,9]. Dispersion relations of some discontinuous Galerkin (DG) methods were obtained in [2]. Here as well as in [17], the dispersive and dissipative errors of DG methods applied to linear advection were studied. In an earlier work [15], the dispersive and dissipative properties of DG methods were analysed in the context of the one-dimensional scalar advection equation as well as the two-dimensional wave equation. This was followed by [3], where the dispersive and dissipative errors of the Interior Penalty Discontinuous Galerkin (IP-DG) method applied to the second-order wave equation were studied. They also studied a more general family of schemes applied to the corresponding first order system. A dispersion analysis of the IP-DG method applied to elastic wave propagation was conducted in [8]. More recently, dispersion analysis of other non-conforming methods have received attention. This includes dispersion analysis of the DPG (discontinuous Petrov-Galerkin) method [13] and the PWDG (plane wave discontinuos Galerkin) method [11].

The purpose of this paper is to use dispersion analysis to quantify the wavenumber discrepancies of certain HDG (Hybrid Discontinuous Galerkin) methods. The HDG method was discovered in [6] and have been applied by many to solve the Helmholtz system - see e.g., $[7,14,10]$. The first work to 
Table 1 Summary of convergence rates of wavenumber errors from [12] (first 3 rows) and this paper (remaining rows). The "0"s indicate that the errors observed were close to machine precision. The row in bold shows SFH rates comparable to the mixed HRT method. All the stated rates are based on observations for $p=0,1,2,3$.

\begin{tabular}{ccccc}
\hline Method & $\tau$ & \multicolumn{3}{c}{ Rates } \\
\cline { 3 - 5 } & & Dispersive error & Dissipative error & Total error \\
\hline LDG-H & 1 & $2 p+3$ & $2 p+2$ & $2 p+2$ \\
LDG-H & $\sqrt{3} i / 2$ & $2 p+2$ & 0 & $2 p+2$ \\
HRT & - & $2 p+3$ & 0 & $2 p+3$ \\
\hline SFH & 1 & $2 p+3$ & $2 p+2$ & $2 p+2$ \\
SFH & $i$ & $2 p+2$ & 0 & $2 p+2$ \\
SFH & $\boldsymbol{i} / \boldsymbol{k} \boldsymbol{h}$ & $\mathbf{2 p}+\mathbf{3}$ & $\mathbf{0}$ & $\mathbf{2 p + 3}$ \\
SFH & $1 / k h$ & $2 p+3$ & $2 p+3$ & $2 p+3$ \\
LDG-H & $i$ & $2 p+2$ & 0 & $2 p+2$ \\
LDG-H & 1 & $2 p+3$ & $2 p+2$ & $2 p+2$ \\
LDG-H & $i / k h$ & $2 p+1$ & 0 & $2 p+1$ \\
LDG-H & $1 / k h$ & $2 p+1$ & $2 p+1$ & $2 p+1$ \\
\hline
\end{tabular}

perform a dispersion analysis of an HDG method was [12]. That work considered one of the standard HDG methods, namely what is called the LDG-H method in [6], studied the influence of its stabilization parameter $\tau$ on the dispersion errors, and compared the HDG errors to those of the mixed HRT (hybrid Raviart-Thomas) method. The results in [12] were limited to the lowest order case $(p=0)$ and the next higher order case $(p=1)$. Based on these, the convergence rate of wavenumber errors when $\tau=1$ was estimated to be $\left|k h-k^{h} h\right|=O(k h)^{2 p+2}$. This, together with other rates are summarized in the first three rows of Table 1 . In a later section, we will confirm the abovementioned LDG-H rates for higher degrees $p$ also.

The results of [12] made it clear that the HRT method surpassed the LDG$\mathrm{H}$ method by yielding higher order wavenumber errors as $h \rightarrow 0$. Therefore, it was natural to ask if any other flavor of the HDG method can possibly achieve rates comparable to the HRT method. The main result of this paper is that the SFH (Single Face HDG) method does indeed exhibit rates comparable to the HRT method for a particular choice of $\tau$. The SFH method uses exactly the same stabilization as the LDG-H method, but applies it in such a way that $\tau=0$ in all but one edge of every mesh element. The SFH method was one of the first HDG methods to be analyzed [5]. Its construction and analysis was motivated by [6] (even though [5] was published before [6]). Due to its 'minimal' stabilization domain, one may view the SFH method as the HDG method that is in some sense 'closest' to the mixed method.

The particular nonzero value of $\tau$ to be specified on the single facet to obtain rates comparable to the HRT method is displayed, together with the accompanying convergence rate, in the last row of Table 1 . The rates obtained with other commonly used values of $\tau$ are also given in Table 1 for comparison. Table 1 thus summarizes the results of this paper and compares it with the previously known results of [12], extrapolating from the limited set of values of $p$ studied. 
In the next section, we introduce HDG methods quickly. In Section 3, we focus on the lowest order SFH method (where $p=0$ ) and perform the dispersion analysis analytically. We are able to obtain an expansion of $k^{h} h-k h$ in closed form in terms of powers of $k h$. This lowest order expansion then suggests the value of $\tau$ that gives higher order convergence. In Section 4, we consider the high order case. We are able to compute $k^{h}$ by numerically solving a nonlinear system (even if we are not able to find an analytical expansion). The same value of $\tau$ that gave the higher rate in the $p=0$ case is found to give a higher rate also for higher $p$.

\section{The HDG method}

Let $\left\{\mathcal{T}_{h}\right\}_{h>0}$ be a family triangulations of the domain $\Omega$ and denote by $\mathcal{E}_{h}$ the set of all the edges of a triangulation $\mathcal{T}_{h}$. Let $\mathbf{n}_{K}$ be the outward unit normal of a triangle $K$, writing $\mathbf{n}$ instead of $\mathbf{n}_{K}$ when there is no confusion. In addition, for a given domain $D$, let $\mathcal{P}_{p}(D)$ denote the space of polynomials of degree at most $p$ defined on $D$. Next, define the following discrete spaces:

$$
\begin{aligned}
V_{h} & =\left\{\mathbf{v} \in\left[L^{2}(\Omega)\right]^{2}:\left.\mathbf{v}\right|_{K} \in\left[\mathcal{P}_{p}(K)\right]^{2}, \forall K \in \mathcal{T}_{h}\right\}, \\
W_{h} & =\left\{\psi \in L^{2}(\Omega):\left.\psi\right|_{K} \in \mathcal{P}_{p}(K), \forall K \in \mathcal{T}_{h}\right\}, \\
M_{h} & =\left\{\widehat{\psi} \in L^{2}\left(\mathcal{E}_{h}\right):\left.\widehat{\psi}\right|_{e} \in \mathcal{P}_{p}(e), \forall e \in \mathcal{E}_{h} \text { and }\left.\widehat{\psi}\right|_{\partial \Omega}=0\right\} .
\end{aligned}
$$

The standard HDG method, i.e., the LDG-H method [6], produces an approximation $\left(\mathbf{u}_{h}, \phi_{h}, \widehat{\phi}_{h}\right)$ of the exact solution $\left(\mathbf{u}, \phi,\left.\phi\right|_{\varepsilon_{h}}\right)$ in the space $V_{h} \times$ $W_{h} \times M_{h}$ that satisfies

$$
\begin{aligned}
\sum_{K \in \mathcal{T}_{h}} i k\left(\mathbf{u}_{h}, \mathbf{v}_{h}\right)_{K}-\left(\phi_{h}, \boldsymbol{\nabla} \cdot \mathbf{v}_{h}\right)_{K}+\left\langle\widehat{\phi}_{h}, \mathbf{v}_{h} \cdot \mathbf{n}\right\rangle_{\partial K} & =0 \\
\sum_{K \in \mathcal{T}_{h}}\left(\boldsymbol{\nabla} \cdot \mathbf{u}_{h}, \psi_{h}\right)_{K}+\left\langle\tau\left(\phi_{h}-\widehat{\phi}_{h}\right), \psi_{h}\right\rangle_{\partial K}+i k\left(\phi_{h}, \psi_{h}\right)_{K} & =\left(f, \psi_{h}\right)_{\Omega}, \\
\sum_{K \in \mathcal{T}_{h}}\left\langle\mathbf{u}_{h} \cdot \mathbf{n}+\tau\left(\phi_{h}-\widehat{\phi}_{h}\right), \widehat{\psi}_{h}\right\rangle_{\partial K} & =0
\end{aligned}
$$

for all $\mathbf{v}_{h} \in V_{h}, \psi_{h} \in W_{h}$ and $\widehat{\psi}_{h} \in M_{h}$, where $\tau$ is a nonzero stabilization function defined on $\partial \mathcal{T}_{h}:=\left\{\partial K: K \in \mathcal{T}_{h}\right\}$. Here $(\cdot, \cdot)_{K}$ and $\langle\cdot, \cdot\rangle_{\partial K}$ denote the inner products of $L^{2}(K)$ and $L^{2}(\partial K)$, respectively. It is traditional to choose $\tau \geq 0$, but for wave problems, as noted in [12], it may often be advantageous to choose $\tau$ in the complex plane. Hence, we will not require that $\tau$ is nonnegative.

Let $\tau_{K}$ denote the value of $\tau$ on $\partial K$. While $\tau_{K}: \partial K \rightarrow \mathbb{C}$ is a single-valued function on each $K$, note that on each edge shared by two triangular elements, $\tau$ is generally double valued. We shall assume that the restriction of $\tau_{K}$ to each edge of $K$ is a constant function. When $\tau_{K}$, for every $K$ in $\mathcal{T}_{h}$, is such that $\tau_{K}$ is zero on all edges except one, we obtain the $\mathrm{SFH}$ method. 
In the next section, we will work with the condensed form of the method. The HDG method was designed specifically to have this condensability feature. The condensed form is obtained by statically condensing out $\mathbf{u}_{h}$ and $\phi_{h}$ to get a single equation for the interface variable $\hat{\phi}_{h}$. We refer to [6] for a general description of this form. We will focus on the condensed form of the lowest order SFH method next.

\section{Dispersion analysis of the lowest order SFH method}

In order to perform the dispersion analysis of the method (2), we follow the approach laid out in $[9,12]$. Consider an infinite triangulation made of isosceles right triangles $K$ with hypotenuse of length $\sqrt{2} h$ and vertical and horizontal edges of length $h$, as shown in Fig. 1. On this infinite grid, we will now derive the condensed form.

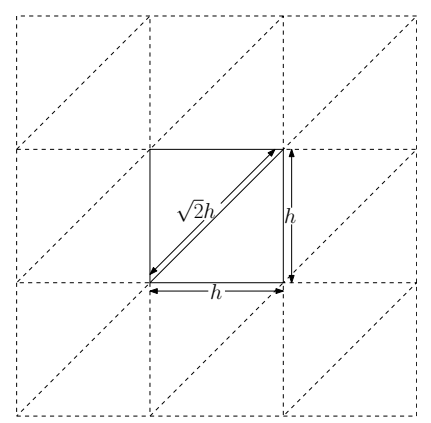

Fig. 1 Sketch of the mesh.

\subsection{Condensed element matrix}

We consider the SFH method associated to (2), where the stabilization parameter $\tau$ is taken to be zero on all vertical and horizontal edges of every triangle. We first analyze the lowest order case $(p=0)$. As we shall see, it is possible to obtain a series expansion of the wavenumber error in closed form in the $p=0$ case. Later, we shall numerically study the high order cases.

Let $K$ be a triangle with edges $e_{1}$ (hypotenuse), $e_{2}$ (horizontal) and $e_{3}$ (vertical). For the polynomial spaces $\left[\mathcal{P}_{0}(K)\right]^{2}, \mathcal{P}_{0}(K), \mathcal{P}_{0}\left(e_{1}\right), \mathcal{P}_{0}\left(e_{2}\right)$ and $\mathcal{P}_{0}\left(e_{3}\right)$, we consider the Dubiner basis functions (see [16]), which for the lowest order case are given by

$$
\mathbf{u}_{1}=\left[\begin{array}{c}
\sqrt{2} \\
0
\end{array}\right], \mathbf{u}_{2}=\left[\begin{array}{c}
0 \\
\sqrt{2}
\end{array}\right], \phi_{0}=\sqrt{2}, \widehat{\phi}_{1}=1, \widehat{\phi}_{2}=1, \text { and }, \widehat{\phi}_{3}=1,
$$


respectively. For elements with the orientation of $K_{1}$ displayed in Fig. 3, the element matrix associated to (2), in the $\left[\mathbf{u}_{1}, \mathbf{u}_{2}, \phi_{0}, \widehat{\phi}_{1}, \widehat{\phi}_{2}, \widehat{\phi}_{3}\right]$-ordering, can be written in block form as

$$
M=\left[\begin{array}{ll}
M_{11} & M_{12} \\
M_{21} & M_{22}
\end{array}\right]
$$

where

$$
\begin{aligned}
M_{11}=\left[\begin{array}{ccc}
i k h^{2} & 0 & 0 \\
0 & i k h^{2} & 0 \\
0 & 0 & -\left(i k h^{2}+2 \sqrt{2} \tau h\right)
\end{array}\right], & M_{12}=\left[\begin{array}{ccc}
\sqrt{2} h & 0 & -\sqrt{2} h \\
-\sqrt{2} h \sqrt{2} h & 0 \\
2 \tau h & 0 & 0,
\end{array}\right], \\
M_{21}=M_{12}^{T}, & M_{22}=\left[\begin{array}{ccc}
-\sqrt{2} \tau h & 0 \\
0 & 0 & 0 \\
0 & 0 & 0
\end{array}\right] .
\end{aligned}
$$

For elements with the orientation of $K_{2}$, also displayed in Fig. 3, the matrices $M_{11}$ and $M_{22}$ are the same, whereas due to the change of the normal vectors, the matrix $M_{12}$ is given by

$$
M_{12}=\left[\begin{array}{ccc}
-\sqrt{2} h & 0 & \sqrt{2} h \\
\sqrt{2} h & -\sqrt{2} h & 0 \\
2 \tau h & 0 & 0
\end{array}\right],
$$

and $M_{21}=M_{12}^{T}$.

The condensation of all interior degrees of freedom is accomplished by taking the Schur complement $S=M_{22}-M_{21} M_{11}^{-1} M_{12}$. In both cases (of $K_{1}$ and $K_{2}$ ), the Schur complement is given by the matrix

$$
S=\left[\begin{array}{rrr}
\frac{4 \tau^{2} h}{i k h+2 \sqrt{2} \tau}+\frac{4 i}{k}-\sqrt{2} \tau h & \frac{-2 i}{k} & \frac{-2 i}{k} \\
\frac{-2 i}{k} & \frac{2 i}{k} & 0 \\
\frac{-2 i}{k} & 0 & \frac{2 i}{k}
\end{array}\right],
$$

a $3 \times 3$ matrix corresponding to the three interface (non-condensable) degrees of freedom, one per edge. Note that while performing this calculation, we have assumed that $M_{11}$ is invertible, which is equivalent to assuming that

$$
2 \sqrt{2} \tau+i k h \neq 0 .
$$

We proceed with the derivation of the dispersion relations by making this assumption throughout. 
3.2 Dispersion relation in the lowest order case

The above-mentioned infinite triangulation has three different types of interface degrees of freedom: the ones associated to the diagonal edges (first type), the horizontal edges (second type) and the vertical edges (third type), as displayed in Fig. 2. We denote by $C_{1}, C_{2}$ and $C_{3}$ the infinite set of stencil centers for the three types of stencils present in this case. Then, we obtain an infinite system of equations for the numerical trace values $\widehat{\phi}_{1, \mathbf{p}_{1}}, \widehat{\phi}_{2, \mathbf{p}_{2}}$ and $\widehat{\phi}_{3, \mathbf{p}_{3}}$ at all $\mathbf{p}_{1} \in C_{1}, \mathbf{p}_{2} \in C_{2}$ and $\mathbf{p}_{3} \in C_{3}$. Since we are interested in how this infinite system propagates plane wave solutions, we consider the ansatz

$$
\widehat{\phi}_{j, \mathbf{p}_{j}}=a_{j} \exp \left(i \mathbf{k}^{h} \cdot \mathbf{p}_{j}\right)
$$

where $a_{1}, a_{2}$ and $a_{3}$ are constants and the components of discrete wave vector $\mathbf{k}^{h}=\left(k_{1}^{h}, k_{2}^{h}\right)$ are given by $k_{1}^{h}:=k^{h} \cos (\theta)$ and $k_{2}^{h}:=k^{h} \sin (\theta)$. Here $k^{h}$ is the unknown discrete wavenumber corresponding to the exact wavenumber $k$, and $\theta$ is the angle of propagation of the plane wave solution. We now proceed to determine an expression that relates $k^{h}$ and $k$. Since we have three types of degrees of freedom, we need to construct three equations, but we will only explain the details of the construction of the first one, associated to diagonal edges: Let's denote by $\mathbf{e}_{1}$ and $\mathbf{e}_{2}$ the cartesian vectors, and suppose that the point $P$ is located at the position $\mathbf{p}_{1}$.

Then, as seen in Fig. 3, the relative position of the point $P_{12}$ (the location in $K_{1}$ of the degree of freedom of the second type) from $P$ is $\mathbf{p}_{1}+\frac{h}{2} \mathbf{e}_{2}$, and the relative position of the point $P_{13}$ (the location in $K_{1}$ of the degree of freedom of the third type) from $P$ is $\mathbf{p}_{1}-\frac{h}{2} \mathbf{e}_{1}$. Hence, the contribution of $K_{1}$ to the first equation is given by

$$
S_{11} \widehat{\phi}_{1, \mathbf{p}_{1}}+S_{12} \widehat{\phi}_{2, \mathbf{p}_{1}+\frac{h}{2} \mathbf{e}_{2}}+S_{13} \widehat{\phi}_{3, \mathbf{p}_{1}-\frac{h}{2} \mathbf{e}_{1}} .
$$

Proceeding in the same way, the contribution of $K_{2}$ to the same equation is given by

$$
S_{11} \widehat{\phi}_{1, \mathbf{p}_{1}}+S_{12} \widehat{\phi}_{2, \mathbf{p}_{1}-\frac{h}{2} \mathbf{e}_{2}}+S_{13} \widehat{\phi}_{3, \mathbf{p}_{1}+\frac{h}{2} \mathbf{e}_{1}} \text {. }
$$

Since plane waves are exact solutions to the Helmholtz equation with zero sources, the right hand side $(f)$ of the equation is zero. Thus, we can write the

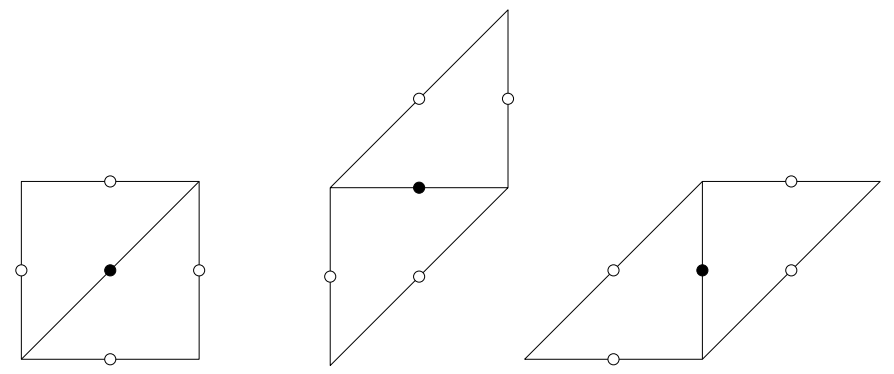

Fig. 2 The three types of stencils for $p=0$. 


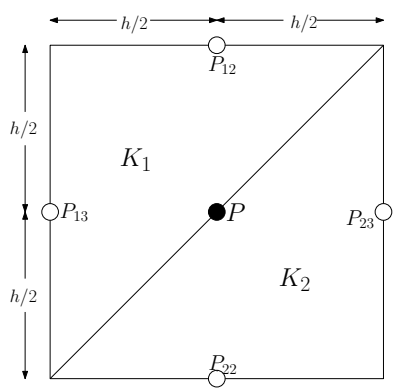

Fig. 3 Positions of the degrees of freedom in the stencil of the first type.

first equation as

$$
\begin{aligned}
2 S_{11} \widehat{\phi}_{1, \mathbf{p}_{1}} & +S_{12}\left(\widehat{\phi}_{2, \mathbf{p}_{1}+\frac{h}{2} \mathbf{e}_{2}}+\widehat{\phi}_{2, \mathbf{p}_{1}-\frac{h}{2} \mathbf{e}_{2}}\right) \\
& +S_{13}\left(\widehat{\phi}_{3, \mathbf{p}_{1}-\frac{h}{2} \mathbf{e}_{1}}+\widehat{\phi}_{3, \mathbf{p}_{1}+\frac{h}{2} \mathbf{e}_{1}}\right)=0 .
\end{aligned}
$$

Since $\widehat{\phi}_{j, \mathbf{p}_{j}}$ is given by (4) and

$$
\widehat{\phi}_{i, \mathbf{p}_{j} \pm \frac{h}{2} \mathbf{e}_{l}}=a_{i} \exp \left(i \mathbf{k}^{h} \cdot\left(\mathbf{p}_{j} \pm \frac{h}{2} \mathbf{e}_{l}\right)\right),
$$

we observe that we can simplify (5) to get

$$
\begin{aligned}
0= & 2 S_{11} a_{1}+S_{12}\left\{\exp \left(\frac{h i}{2} \mathbf{k}^{h} \cdot \mathbf{e}_{2}\right)+\exp \left(-\frac{h i}{2} \mathbf{k}^{h} \cdot \mathbf{e}_{2}\right)\right\} a_{2} \\
& +S_{13}\left\{\exp \left(-\frac{h i}{2} \mathbf{k}^{h} \cdot \mathbf{e}_{1}\right)+\exp \left(\frac{h i}{2} \mathbf{k}^{h} \cdot \mathbf{e}_{1}\right)\right\} a_{3} \\
= & 2 S_{11} a_{1}+S_{12}\left\{\exp \left(\frac{h i}{2} k^{h} \sin (\theta)\right)+\exp \left(-\frac{h i}{2} k^{h} \sin (\theta)\right)\right\} a_{2} \\
& +S_{13}\left\{\exp \left(-\frac{h i}{2} k^{h} \cos (\theta)\right)+\exp \left(\frac{h i}{2} k^{h} \cos (\theta)\right)\right\} a_{3} .
\end{aligned}
$$

Using that $\exp (i \alpha)=\cos (\alpha)+i \sin (\alpha)$ for any $\alpha \in \mathbb{R}$, and some well known properties of complex numbers, we can write our equation as

$$
2 S_{11} a_{1}+2 S_{12} \cos \left(\frac{h}{2} k^{h} \cos (\theta)\right) a_{2}+2 S_{13} \cos \left(\frac{h}{2} k^{h} \sin (\theta)\right) a_{3}=0 .
$$

Finally, substituting

$$
S_{11}=\frac{4 \tau^{2} h}{i k h+2 \sqrt{2} \tau}+\frac{4 i}{k}-\sqrt{2} \tau h, \quad S_{12}=S_{13}=\frac{-2 i}{k},
$$

multiplying the equation by $\frac{k}{2}(i k h+2 \sqrt{2} \tau)$, and rearranging terms, we obtain

$$
\begin{aligned}
\left\{(4 i-\sqrt{2} \tau k h)(i k h+2 \sqrt{2} \tau)+4 \tau^{2} k h\right\} a_{1} & -2 i(i k h+2 \sqrt{2} \tau) c_{2} a_{2} \\
& -2 i(i k h+2 \sqrt{2} \tau) c_{1} a_{3}=0
\end{aligned}
$$


where $c_{j}=\cos \left(h k_{j}^{h} / 2\right), j=1,2$, and

$$
k_{1}^{h}=k^{h} \cos (\theta), \quad k_{2}^{h}=k^{h} \sin (\theta) .
$$

This is the equation for the first type of degrees of freedom. The other two equations may be derived in the same way.

The three equations together form a system

$$
F\left[\begin{array}{l}
a_{1} \\
a_{2} \\
a_{3}
\end{array}\right]=0
$$

where $F$ is the so-called dispersion matrix, given by

$F=\left[\begin{array}{ccc}(4 i-\sqrt{2} \tau k h)(i k h+2 \sqrt{2} \tau)+4 \tau^{2} k h-2 i(i k h+2 \sqrt{2} \tau) c_{2}-2 i(i k h+2 \sqrt{2} \tau) c_{1} \\ -c_{2} & 1 & 0 \\ -c_{1} & 0 & 1\end{array}\right]$.

Its determinant is

$$
\operatorname{det}(F)=-2 i(2 \sqrt{2} \tau+i k h)\left(c_{1}^{2}+c_{2}^{2}\right)+(2 \sqrt{2} \tau+i k h)(4 i-\sqrt{2} \tau k h)+4 \tau^{2} k h .
$$

We are interested in non-trivial solutions of this system. Hence we conclude that the dispersion relation relating $k^{h}$ to $k$ in the $\mathrm{SFH}$ method is

$$
\operatorname{det}(F)=0
$$

Note that since the entries of $F$ depend on $k^{h}$, the above forms a nonlinear equation for the discrete wavenumber $k^{h}$.

\subsection{Asymptotic expansion of the wavenumber error}

To get useful qualitative information about $k^{h}$, we must further manipulate (7). By virtue of (3), we may multiply (7) by $-[2 i(2 \sqrt{2} \tau+i k h)]^{-1}$ and simplify to obtain

$$
c_{1}^{2}+c_{2}^{2}+\frac{i\left\{(2 \sqrt{2} \tau+i k h)(4 i-\sqrt{2} \tau k h)+4 \tau^{2} k h\right\}}{2(2 \sqrt{2} \tau+i k h)}=0
$$

The identity $i\left\{(2 \sqrt{2} \tau+i k h)(4 i-\sqrt{2} \tau k h)+4 \tau^{2} k h\right\}=\left(\sqrt{2}(k h)^{2}-8 \sqrt{2}\right) \tau-$ $4 i k h$ now motivates us to set

$$
R=-\frac{\left(\sqrt{2}(k h)^{2}-8 \sqrt{2}\right) \tau-4 i k h}{2(2 \sqrt{2} \tau+i k h)},
$$


and rewrite (8) as

$$
\begin{aligned}
0 & =c_{1}^{2}+c_{2}^{2}-R \\
& =\left(c_{1}^{2}-1\right)+\left(c_{2}^{2}-1\right)+(2-R) \\
& =\left(c_{1}^{2}-1\right)+\left(c_{2}^{2}-1\right)+(2-R)\left(\cos ^{2} \theta+\sin ^{2} \theta\right) \\
& =\left\{\left(c_{1}^{2}-1\right)+(2-R) \cos ^{2}(\theta)\right\}+\left\{\left(c_{2}^{2}-1\right)+(2-R) \sin ^{2}(\theta)\right\} .
\end{aligned}
$$

Thus, we identify two sufficient conditions for $\operatorname{det}(F)=0$ to hold, namely

$$
c_{1}=\left(1-d_{1}\right)^{1 / 2}, \quad c_{2}=\left(1-d_{2}\right)^{1 / 2},
$$

where $d_{1}=(2-R) \cos ^{2}(\theta)$ and $d_{2}=(2-R) \sin ^{2}(\theta)$.

The two sufficient conditions take similar forms. As we shall see now, they are simple enough to understand by Taylor expansion. In analogy with (6), let

$$
k_{1}=k \cos (\theta), \quad k_{2}=k \sin (\theta) .
$$

By (9) and the definitions of $c_{j}, d_{j}$, we have

$$
k_{j}^{h} h=2 \cos ^{-1}\left(\sqrt{1-d_{j}}\right), \quad d_{j}=\frac{\sqrt{2} \tau\left(k_{j} h\right)^{2}}{4 \sqrt{2} \tau+2 i(k h)} .
$$

As $k h \rightarrow 0$, clearly $d_{j} \rightarrow 0$, so using the Taylor expansion of arccosine near 1 , we obtain

$$
\begin{aligned}
& k_{1}^{h} h=\cos (\theta)(k h)-\frac{i \sqrt{2} \cos (\theta)}{8 \tau}(k h)^{2}+\frac{8 \tau^{2} \cos ^{3}(\theta)-9 \cos (\theta)}{192 \tau^{2}}(k h)^{3}+O(k h)^{4} \\
& k_{2}^{h} h=\sin (\theta)(k h)-\frac{i \sqrt{2} \sin (\theta)}{8 \tau}(k h)^{2}+\frac{8 \tau^{2} \sin ^{3}(\theta)-9 \sin (\theta)}{192 \tau^{2}}(k h)^{3}+O(k h)^{4}
\end{aligned}
$$

These relations, together with $k^{h}=\left(k_{1}^{h}\right)^{2}+\left(k_{2}^{h}\right)^{2}$, yield

$$
k^{h} h-k h=\frac{i}{4 \sqrt{2} \tau}(k h)^{2}+\frac{2 \tau^{2} \cos (4 \theta)+6 \tau^{2}-9}{192 \tau^{2}}(k h)^{3}+O(k h)^{4}
$$

as $k h \rightarrow 0$. This is the main result of this subsection.

The series expansion (10) immediately shows that the wavenumber error is of order 2 (equalling $2 p+2$ when $p=0$ ) for any nonzero $\tau$. Next, note how $\tau$ appears in the first and second terms of the expansion (10). In the first term, it only appears in the denominator (while in the second term it appears in equal degrees in the denominator and numerator). This leads us to an important observation: If we set

$$
\tau=\frac{i}{k h}
$$

in (10), then we should obtain the higher rate of convergence $\left|k^{h} h-k h\right|=$ $O(k h)^{3}$ as $k h \rightarrow 0$. Moreover, we see that all terms in the series expansion of $k^{h} h-k h$, except the first, have even powers of $\tau$ so $\operatorname{Im}\left(k^{h} h-k h\right)=0$, if $\tau$ is set by (11) or any real multiple of it, i.e., the dissipative errors should vanish. We will gather numerical confirmation of these statements in the next subsection. 
Table 2 Results for $\tau=i$ and $\tau=1$ in the lowest order SFH method.

\begin{tabular}{l||cc|cc|cc||cc|cc|cc}
\multicolumn{1}{l||}{} & \multicolumn{9}{c||}{$\tau=i$} \\
$k h$ & $\epsilon_{\text {disp }}$ & rate & $\epsilon_{\text {dissip }}$ & rate & $\epsilon_{\text {total }}$ & rate & $\epsilon_{\text {disp }}$ & rate & $\epsilon_{\text {dissip }}$ & rate & $\epsilon_{\text {total }}$ & rate \\
\hline$\pi / 2^{2}$ & $8.34 \mathrm{e}-02$ & - & $4.42 \mathrm{e}-16$ & - & $8.34 \mathrm{e}-02$ & - & $1.25 \mathrm{e}-02$ & - & $1.13 \mathrm{e}-01$ & - & $1.13 \mathrm{e}-01$ & - \\
$\pi / 2^{3}$ & $2.37 \mathrm{e}-02$ & 1.82 & $7.56 \mathrm{e}-16$ & - & $2.37 \mathrm{e}-02$ & 1.82 & $1.57 \mathrm{e}-03$ & 2.99 & $2.75 \mathrm{e}-02$ & 2.03 & $2.75 \mathrm{e}-02$ & 2.04 \\
$\pi / 2^{4}$ & $6.34 \mathrm{e}-03$ & 1.90 & $1.03 \mathrm{e}-15$ & - & $6.34 \mathrm{e}-03$ & 1.90 & $1.97 \mathrm{e}-04$ & 3.00 & $6.83 \mathrm{e}-03$ & 2.01 & $6.83 \mathrm{e}-03$ & 2.01 \\
$\pi / 2^{5}$ & $1.64 \mathrm{e}-03$ & 1.95 & $2.90 \mathrm{e}-15$ & - & $1.64 \mathrm{e}-03$ & 1.95 & $2.46 \mathrm{e}-05$ & 3.00 & $1.70 \mathrm{e}-03$ & 2.00 & $1.70 \mathrm{e}-03$ & 2.00 \\
$\pi / 2^{6}$ & $4.18 \mathrm{e}-04$ & 1.97 & $9.54 \mathrm{e}-15$ & - & $4.18 \mathrm{e}-04$ & 1.97 & $3.08 \mathrm{e}-06$ & 3.00 & $4.26 \mathrm{e}-04$ & 2.00 & $4.26 \mathrm{e}-04$ & 2.00 \\
$\pi / 2^{7}$ & $1.05 \mathrm{e}-04$ & 1.99 & $1.16 \mathrm{e}-14$ & - & $1.05 \mathrm{e}-04$ & 1.99 & $3.85 \mathrm{e}-07$ & 3.00 & $1.06 \mathrm{e}-04$ & 2.00 & $1.06 \mathrm{e}-04$ & 2.00 \\
$\pi / 2^{8}$ & $2.65 \mathrm{e}-05$ & 1.99 & $3.55 \mathrm{e}-14$ & - & $2.65 \mathrm{e}-05$ & 1.99 & $4.81 \mathrm{e}-08$ & 3.00 & $2.66 \mathrm{e}-05$ & 2.00 & $2.66 \mathrm{e}-05$ & 2.00 \\
$\pi / 2^{9}$ & $6.64 \mathrm{e}-06$ & 2.00 & $2.92 \mathrm{e}-14$ & - & $6.64 \mathrm{e}-06$ & 2.00 & $6.02 \mathrm{e}-09$ & 3.00 & $6.66 \mathrm{e}-06$ & 2.00 & $6.66 \mathrm{e}-06$ & 2.00 \\
$\pi / 2^{10}$ & $1.66 \mathrm{e}-06$ & 2.00 & $1.96 \mathrm{e}-13$ & - & $1.66 \mathrm{e}-06$ & 2.00 & $7.52 \mathrm{e}-10$ & 3.00 & $1.66 \mathrm{e}-06$ & 2.00 & $1.66 \mathrm{e}-06$ & 2.00
\end{tabular}

3.4 Numerical computation of $k^{h}$ for the lowest order SFH method

Since the above-derived error expansion is only asymptotic, we now proceed to check the practical size of wavenumber errors by direct numerical computation. We solve the nonlinear dispersion relation (7) numerically to find $k^{h}$. Recall that $k^{h} \equiv k^{h}(\theta)$ is a function of the propagation angle $\theta$. In all the cases we present below, we consider $\theta \in \Theta=\{j \pi / 40: j=1, \ldots, 20\}$. The exact wavenumber is set so that $k h \in\left\{\pi / 2^{j+2}: j=0, \ldots, 8\right\}$. (Note that the dependence on $k$ occurs in the form of dependence on $k h$ in the dispersion relations.) Define

$$
\begin{gathered}
\epsilon_{\text {disp }}=\max _{\theta \in \Theta}\left|\operatorname{Re}\left(k^{h}(\theta) h\right)-k h\right|, \quad \epsilon_{\text {dissip }}=\max _{\theta \in \Theta}\left|\operatorname{Im}\left(k^{h}(\theta) h\right)\right|, \\
\epsilon_{\text {total }}=\max _{\theta \in \Theta}\left|k^{h}(\theta)-k h\right| .
\end{gathered}
$$

We report the numerically computed values of these numbers for the following cases.

Case 1: $\tau=i$. Equation (7) can now be simplified to the form

$$
\operatorname{det}(F)=2(2 \sqrt{2}+k h)\left(c_{1}^{2}+c_{2}^{2}\right)-(2 \sqrt{2}+k h)(4-\sqrt{2} k h)-4 k h=0 .
$$

This is the form used in our nonlinear solve. For small $k h$, equation specifies values of $c_{1}^{2}+c_{2}^{2}$ that admit real solutions for $k^{h}$. The values of $k^{h}$ found by the numerical root finder, reported in Table 2, confirms that $\epsilon_{\text {dissip }}$ is 0 up to machine precision. The total error $\epsilon_{\text {total }}$ appears to go to 0 at the rate $O(k h)^{2}$, as expected from the asymptotic expansion (10).

Case 2: $\tau=1$. In this case the determinant simplifies to

$$
\operatorname{det}(F)=\left\{2 k h\left(c_{1}^{2}+c_{2}^{2}\right)-4 k\right\}+i\left\{-4 \sqrt{2}\left(c_{1}^{2}+c_{2}^{2}\right)+8 \sqrt{2}-2(k h)^{2}\right\},
$$

and its no longer clear that solutions of the dispersion relation are real. Searching for roots in the complex plane, we computed $k^{h}$. These results are compiled in Table 2. Clearly, the dissipative errors seem to dominate in this case. As before, $\epsilon_{\text {total }}=O(k h)^{2}$. 
Table 3 Results for $\tau=\frac{i}{k h}$ and $\tau=\frac{1}{k h}$ in the lowest order SFH method.

\begin{tabular}{l||cc|cc|cc||cc|cc|cc}
\multicolumn{1}{l||}{} & \multicolumn{9}{c||}{$\tau=\frac{c}{k h}$} & \multicolumn{5}{c}{$\tau=\frac{1}{k h}$} \\
$k h$ & $\epsilon_{\text {disp }}$ & rate & $\epsilon_{\text {dissip }}$ & rate & $\epsilon_{\text {total }}$ & rate & $\epsilon_{\text {disp }}$ & rate & $\epsilon_{\text {dissip }}$ & rate & $\epsilon_{\text {total }}$ & rate \\
\hline$\pi / 2^{2}$ & $6.60 \mathrm{e}-02$ & - & $6.09 \mathrm{e}-16$ & - & $6.60 \mathrm{e}-02$ & - & $6.18 \mathrm{e}-03$ & - & $9.01 \mathrm{e}-02$ & - & $9.03 \mathrm{e}-02$ & - \\
$\pi / 2^{3}$ & $9.11 \mathrm{e}-03$ & 2.86 & $1.40 \mathrm{e}-15$ & - & $9.11 \mathrm{e}-03$ & 2.86 & $2.12 \mathrm{e}-03$ & 1.55 & $1.09 \mathrm{e}-02$ & 3.05 & $1.11 \mathrm{e}-02$ & 3.02 \\
$\pi / 2^{4}$ & $1.17 \mathrm{e}-03$ & 2.96 & $1.60 \mathrm{e}-15$ & - & $1.17 \mathrm{e}-03$ & 2.96 & $3.03 \mathrm{e}-04$ & 2.80 & $1.34 \mathrm{e}-03$ & 3.02 & $1.38 \mathrm{e}-03$ & 3.01 \\
$\pi / 2^{5}$ & $1.47 \mathrm{e}-04$ & 2.99 & $2.79 \mathrm{e}-15$ & - & $1.47 \mathrm{e}-04$ & 2.99 & $3.90 \mathrm{e}-05$ & 2.96 & $1.67 \mathrm{e}-04$ & 3.01 & $1.72 \mathrm{e}-04$ & 3.00 \\
$\pi / 2^{6}$ & $1.84 \mathrm{e}-05$ & 3.00 & $6.20 \mathrm{e}-15$ & - & $1.84 \mathrm{e}-05$ & 3.00 & $4.92 \mathrm{e}-06$ & 2.99 & $2.09 \mathrm{e}-05$ & 3.00 & $2.15 \mathrm{e}-05$ & 3.00 \\
$\pi / 2^{7}$ & $2.31 \mathrm{e}-06$ & 3.00 & $2.42 \mathrm{e}-14$ & - & $2.31 \mathrm{e}-06$ & 3.00 & $6.16 \mathrm{e}-07$ & 3.00 & $2.61 \mathrm{e}-06$ & 3.00 & $2.69 \mathrm{e}-06$ & 3.00 \\
$\pi / 2^{8}$ & $2.88 \mathrm{e}-07$ & 3.00 & $1.29 \mathrm{e}-14$ & - & $2.88 \mathrm{e}-07$ & 3.00 & $7.70 \mathrm{e}-08$ & 3.00 & $3.27 \mathrm{e}-07$ & 3.00 & $3.36 \mathrm{e}-07$ & 3.00 \\
$\pi / 2^{9}$ & $3.60 \mathrm{e}-08$ & 3.00 & $1.89 \mathrm{e}-14$ & - & $3.60 \mathrm{e}-08$ & 3.00 & $9.63 \mathrm{e}-09$ & 3.00 & $4.08 \mathrm{e}-08$ & 3.00 & $4.20 \mathrm{e}-08$ & 3.00 \\
$\pi / 2^{10}$ & $4.50 \mathrm{e}-09$ & 3.00 & $8.42 \mathrm{e}-14$ & - & $4.50 \mathrm{e}-09$ & 3.00 & $1.20 \mathrm{e}-09$ & 3.00 & $5.10 \mathrm{e}-09$ & 3.00 & $5.24 \mathrm{e}-09$ & 3.00
\end{tabular}

Case 3: $\tau=i / k h$. This is the interesting value of $\tau$ found in (11). Here the determinant simplifies to

$$
\operatorname{det}(F)=\left(\frac{2 \sqrt{2}}{k h}+k h\right)\left(2\left(c_{1}^{2}+c_{2}^{2}\right)-4+\sqrt{2}\right)-\frac{4}{k h} .
$$

The results are now in Table 3. As expected from our discussions following the asymptotic expansion (10), the dissipative errors are zero to machine precision and moreover, we obtain the higher rate of convergence of $O(k h)^{3}$ for the total error.

Case 4: $\tau=1 / k h$. This case differs from the previous case only by a scalar multiple. Hence, reviewing the expansion (10), we expect to obtain higher order convergence for the total error in this case also. The determinant in this case is

$$
\operatorname{det}(F)=2\left(\frac{2 \sqrt{2}}{k h}+i k h\right)\left(c_{1}^{2}+c_{2}^{2}\right)-\left(\frac{2 \sqrt{2}}{k h}+i k h\right)(4 i-\sqrt{2})+\frac{4}{k h} .
$$

The results, displayed in Table 3 , show that although $\epsilon_{\text {dissip }}$ is no longer zero, the total error $\epsilon_{\text {total }}$ goes to zero at the faster rate $O(k h)^{3}$.

\section{Wavenumber errors in the higher order SFH method}

In order to go beyond the $p=0$ case, we will use the technique of [12]. The main idea is to obtain an analogue of (7) with a larger matrix $F$. This will then be numerically solved for the discrete wavenumber $k^{h}=k^{h}(\theta)$. As we did in the lowest order case, we will use the same infinite lattice of isosceles right triangles, and the ansatz that the degrees of freedom interpolate a plane wave traveling in the $\theta$ direction with wavenumber $k^{h}$. 
4.1 The dispersion relation

We describe the technique in detail for a method with $L$ different node types. Recall that the lowest order SFH method had three node types. The first order SFH method, with $p=1$, will have six node types. As $p$ increases, $L$ will increase as well. Let us denote the solution value at a node of the $l^{\text {th }}$ type, $1 \leq l \leq L$, located at $\mathbf{r} h \in \mathbb{R}^{2}$, by $\psi_{l, \mathbf{r}}$. With our ansatz that these solution values interpolate a plane wave, we have that

$$
\psi_{l, \mathbf{r}}=a_{l} e^{i \mathbf{k}^{h} \cdot \mathbf{r} h},
$$

for some constants $a_{l}$.

We will now construct the equation of a fixed stencil within the lattice. Suppose that it corresponds to a node of the $t^{t h}$ type, $1 \leq t \leq L$, that is located at $\mathbf{j} h$. For $1 \leq l \leq L$, define the set $J_{t, l}=\left\{\mathbf{r} \in \mathbb{R}^{2}: \mathbf{r}\right.$ is a node of type $s$ located at $(\mathbf{j}+\mathbf{r}) h\}$. For $\mathbf{r} \in J_{t, l}$, denote the stencil coefficient of the node at location $(\mathbf{j}+\mathbf{r}) h$ by $D_{t, l, \mathbf{r}}$. Since plane waves are exact solutions to the Helmholtz equation with zero sources, the stencil's equation is

$$
\sum_{l=1}^{L} \sum_{\mathbf{r} \in J_{t, l}} D_{t, l, \mathbf{r}} \psi_{l, \mathbf{j}+\mathbf{r}}=0 .
$$

Finally, we remove all dependence on $\mathbf{j}$ in this equation by dividing by $e^{i k^{h} \cdot \mathbf{j} h}$, so there are $L$ equations in total, with the $t^{t h}$ equation given by

$$
\sum_{l=1}^{L} a_{l} \sum_{\mathbf{r} \in J_{t, l}} D_{t, l, \mathbf{r}} e^{i \mathbf{k}^{h} \cdot \mathbf{r} h}=0 .
$$

Thus, we can now define the $L \times L$ matrix $F\left(k^{h}\right)$ by

$$
\left[F\left(k^{h}\right)\right]_{t, l}=\sum_{\mathbf{r} \in J_{t, l}} D_{t, l, \mathbf{r}} e^{i k^{h}[\cos (\theta), \sin (\theta)] \cdot \mathbf{r} h},
$$

and observe that (12) has a non-trivial solution $a_{1}, \ldots, a_{L}$ if and only if $k^{h}$ is such that

$$
\operatorname{det}\left(F\left(k^{h}\right)\right)=0 .
$$

This is the analogue of (7) for higher $p$. We need to solve it to determine $k^{h}$ for any given $\theta$.

4.2 Numerical computation of $k^{h}$ for the higher order SFH method.

We will use the same values for the exact wavenumber $k h$ and the angle $\theta$ as in the lowest order case, namely, $\theta \in\{j \pi / 40: j=1, \ldots, 20\}$ and $k h \in$ $\left\{\pi / 2^{j+2}: j=0, \ldots, 8\right\}$. We also measure the errors $\epsilon_{\text {disp }}, \epsilon_{\text {dissip }}$ and $\epsilon_{\text {total }}$, defined before. Due to the increased complexity, we are unable to write down 
Table 4 Results for $\tau=i$ and $\tau=1$ for the SFH method with $p=1$.

\begin{tabular}{l||cc|cc|cc||cc|cc|cc}
\multicolumn{1}{l||}{} & \multicolumn{9}{|c||}{$\tau=i$} \\
$k h$ & $\epsilon_{\text {disp }}$ & rate & $\epsilon_{\text {dissip }}$ & rate & $\epsilon_{\text {total }}$ & rate & $\epsilon_{\text {disp }}$ & rate & $\epsilon_{\text {dissip }}$ & rate & $\epsilon_{\text {total }}$ & rate \\
\hline$\pi / 2^{2}$ & $2.37 \mathrm{e}-03$ & - & $4.96 \mathrm{e}-16$ & - & $2.37 \mathrm{e}-03$ & - & $3.71 \mathrm{e}-04$ & - & $2.69 \mathrm{e}-03$ & - & $2.72 \mathrm{e}-03$ & - \\
$\pi / 2^{3}$ & $1.62 \mathrm{e}-04$ & 3.87 & $1.64 \mathrm{e}-15$ & - & $1.62 \mathrm{e}-04$ & 3.87 & $1.14 \mathrm{e}-05$ & 5.02 & $1.73 \mathrm{e}-04$ & 3.96 & $1.74 \mathrm{e}-04$ & 3.97 \\
$\pi / 2^{4}$ & $1.05 \mathrm{e}-05$ & 3.94 & $3.39 \mathrm{e}-15$ & - & $1.05 \mathrm{e}-05$ & 3.94 & $3.55 \mathrm{e}-07$ & 5.01 & $1.09 \mathrm{e}-05$ & 3.99 & $1.09 \mathrm{e}-05$ & 3.99 \\
$\pi / 2^{5}$ & $6.72 \mathrm{e}-07$ & 3.97 & $5.61 \mathrm{e}-15$ & - & $6.72 \mathrm{e}-07$ & 3.97 & $1.11 \mathrm{e}-08$ & 5.00 & $6.84 \mathrm{e}-07$ & 4.00 & $6.84 \mathrm{e}-07$ & 4.00 \\
$\pi / 2^{6}$ & $4.24 \mathrm{e}-08$ & 3.99 & $8.49 \mathrm{e}-15$ & - & $4.24 \mathrm{e}-08$ & 3.99 & $3.46 \mathrm{e}-10$ & 5.00 & $4.28 \mathrm{e}-08$ & 4.00 & $4.28 \mathrm{e}-08$ & 4.00 \\
$\pi / 2^{7}$ & $2.66 \mathrm{e}-09$ & 3.99 & $1.75 \mathrm{e}-14$ & - & $2.66 \mathrm{e}-09$ & 3.99 & $1.09 \mathrm{e}-11$ & 4.99 & $2.67 \mathrm{e}-09$ & 4.00 & $2.67 \mathrm{e}-09$ & 4.00 \\
$\pi / 2^{8}$ & $1.67 \mathrm{e}-10$ & 4.00 & $3.11 \mathrm{e}-14$ & - & $1.67 \mathrm{e}-10$ & 4.00 & $4.51 \mathrm{e}-13$ & 4.59 & $1.67 \mathrm{e}-10$ & 4.00 & $1.67 \mathrm{e}-10$ & 4.00 \\
$\pi / 2^{9}$ & $1.04 \mathrm{e}-11$ & 4.00 & $8.21 \mathrm{e}-14$ & - & $1.04 \mathrm{e}-11$ & 4.00 & $4.93 \mathrm{e}-13$ & - & $1.04 \mathrm{e}-11$ & 4.00 & $1.05 \mathrm{e}-11$ & 4.00 \\
$\pi / 2^{10}$ & $9.22 \mathrm{e}-13$ & 3.50 & $3.07 \mathrm{e}-13$ & - & $9.72 \mathrm{e}-13$ & - & $6.43 \mathrm{e}-13$ & - & $7.39 \mathrm{e}-13$ & 3.82 & $9.00 \mathrm{e}-13$ & -
\end{tabular}

Table 5 Results for $\tau=\frac{i}{k h}$ and $\tau=\frac{1}{k h}$ for the SFH method with $p=1$.

\begin{tabular}{|c|c|c|c|c|c|c|c|c|c|c|c|c|}
\hline \multirow[b]{2}{*}{$k h$} & \multicolumn{6}{|c|}{$\tau=\frac{i}{k h}$} & \multicolumn{6}{|c|}{$\tau=\frac{1}{k h}$} \\
\hline & $\epsilon_{\text {disp }}$ & rate & $\epsilon_{\text {dissip }}$ & rate & $\epsilon_{\text {total }}$ & rate & $\epsilon_{\text {disp }}$ & rate & $\epsilon_{\text {dissip }}$ & rate & $\epsilon_{\text {total }}$ & rate \\
\hline$\pi / 2^{2}$ & $1.91 \mathrm{e}-03$ & - & $7.29 \mathrm{e}-16$ & - & $1.91 \mathrm{e}-03$ & - & $2.21 \mathrm{e}-04$ & - & $2.13 \mathrm{e}-03$ & - & $2.14 \mathrm{e}-03$ & - \\
\hline$\pi / 2^{3}$ & $6.58 \mathrm{e}-05$ & 4.86 & $2.68 \mathrm{e}-15$ & - & $6.58 \mathrm{e}-05$ & 4.86 & $1.99 \mathrm{e}-06$ & 6.79 & $6.84 \mathrm{e}-05$ & 4.96 & $6.84 \mathrm{e}-05$ & 4.97 \\
\hline$\pi / 2^{4}$ & $2.11 \mathrm{e}-06$ & 4.96 & $4.09 \mathrm{e}-15$ & - & $2.11 \mathrm{e}-06$ & 4.96 & $9.17 \mathrm{e}-08$ & 4.44 & $2.15 \mathrm{e}-06$ & 4.99 & $2.15 \mathrm{e}-06$ & 4.99 \\
\hline$\pi / 2^{5}$ & $6.62 \mathrm{e}-08$ & 4.99 & $7.82 \mathrm{e}-15$ & $\begin{array}{lll}- & - \\
\end{array}$ & $6.62 \mathrm{e}-08$ & 4.99 & $3.09 \mathrm{e}-09$ & 4.89 & $6.72 \mathrm{e}-08$ & 5.00 & $6.72 \mathrm{e}-08$ & 5.00 \\
\hline$\pi / 2^{6}$ & 2.07e-09 & 5.00 & $8.19 \mathrm{e}-15$ & - & $2.07 \mathrm{e}-09$ & 5.00 & $9.84 \mathrm{e}-11$ & 4.97 & $2.10 \mathrm{e}-09$ & 5.00 & $2.10 \mathrm{e}-09$ & 5.00 \\
\hline$\pi / 2^{7}$ & $6.48 \mathrm{e}-11$ & 5.00 & $1.99 \mathrm{e}-14$ & 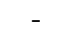 & $6.48 \mathrm{e}-11$ & 5.00 & $3.06 \mathrm{e}-12$ & 5.01 & $6.56 \mathrm{e}-11$ & 5.00 & 6.5 & 5.00 \\
\hline$\pi / 2^{8}$ & $1.95 \mathrm{e}-12$ & 5.05 & $2.33 \mathrm{e}-14$ & - & $1.95 \mathrm{e}-12$ & 5.05 & $1.95 \mathrm{e}-13$ & 3.97 & $2.06 \mathrm{e}-12$ & 4.99 & $2.07 \mathrm{e}-12$ & 4.99 \\
\hline$\pi / 2^{9}$ & $3.56 \mathrm{e}-13$ & - & $4.39 \mathrm{e}-14$ & - & $3.57 \mathrm{e}-13$ & - & $4.05 \mathrm{e}-13$ & - & $8.88 \mathrm{e}-14$ & - & $4.08 \mathrm{e}-13$ & - \\
\hline$\pi / 2^{10}$ & $1.03 \mathrm{e}-12$ & - & $1.60 \mathrm{e}-13$ & - & $1.03 \mathrm{e}-12$ & - & $5.19 \mathrm{e}-13$ & - & 3.31e-13 & - & $5.21 \mathrm{e}-13$ & - \\
\hline
\end{tabular}

analytical expressions for the determinant in each case, as we did in Section 3.4. The results for $p=1,2$ and 3 are shown in Tables $4-5$, Tables $6-7$ and Tables 8 9 , respectively. We continue to consider the standard case of $\tau=1$, in addition to the nonstandard cases of $\tau=i, \tau=i / k h$ and $\tau=1 / k h$. Recall that the latter two cases were motivated by the asymptotic expansion (10) in the lowest order case. The tabulated results show that these choices continue to remain superior in the higher order case also.

Taken together, these results and those of Section 3.4, indicate that for $\tau=i / k h$, the dissipative error is zero, and the total error goes to zero at the rate

$$
\epsilon_{\text {total }}=O(k h)^{2 p+3}, \quad k h \rightarrow 0
$$

for $p=0,1,2$ and 3. For the remaining values of $\tau$ considered, we get convergence of the total error at one less rate. These are the rates we summarized in the introduction (see Table 1). To our knowledge, the SFH method with $\tau=i / k h$ is the only $D G$ method to give such rates comparable to the mixed (HRT) method.

\section{Results for LDG-H method}

In this section we consider (2) with $\tau$ set to the same nonzero positive constant on every edge and numerically explore the relation between $k^{h}$ and $k$. This 
Table 6 Results for $\tau=i$ and $\tau=1$ for the SFH with $p=2$.

\begin{tabular}{l||cc|cc|cc||cc|cc|cc}
\multicolumn{1}{c||}{} & \multicolumn{9}{c||}{$\tau=i$} \\
$k h$ & $\epsilon_{\text {disp }}$ & rate & $\epsilon_{\text {dissip }}$ & rate & $\epsilon_{\text {total }}$ & rate & $\epsilon_{\text {disp }}$ & rate & $\epsilon_{\text {dissip }}$ & rate & $\epsilon_{\text {total }}$ & rate \\
\hline$\pi / 2^{2}$ & $2.35 \mathrm{e}-05$ & - & $1.55 \mathrm{e}-15$ & - & $2.35 \mathrm{e}-05$ & - & $2.67 \mathrm{e}-06$ & - & $2.51 \mathrm{e}-05$ & - & $2.53 \mathrm{e}-05$ & - \\
$\pi / 2^{3}$ & $3.87 \mathrm{e}-07$ & 5.92 & $1.25 \mathrm{e}-15$ & - & $3.87 \mathrm{e}-07$ & 5.92 & $2.11 \mathrm{e}-08$ & 6.98 & $4.02 \mathrm{e}-07$ & 5.97 & $4.03 \mathrm{e}-07$ & 5.97 \\
$\pi / 2^{4}$ & $6.20 \mathrm{e}-09$ & 5.97 & $2.96 \mathrm{e}-15$ & - & $6.20 \mathrm{e}-09$ & 5.97 & $1.66 \mathrm{e}-10$ & 7.00 & $6.32 \mathrm{e}-09$ & 5.99 & $6.32 \mathrm{e}-09$ & 5.99 \\
$\pi / 2^{5}$ & $9.79 \mathrm{e}-11$ & 5.98 & $3.78 \mathrm{e}-15$ & - & $9.79 \mathrm{e}-11$ & 5.98 & $1.29 \mathrm{e}-12$ & 7.00 & $9.89 \mathrm{e}-11$ & 6.00 & $9.89 \mathrm{e}-11$ & 6.00 \\
$\pi / 2^{6}$ & $1.50 \mathrm{e}-12$ & 6.03 & $1.41 \mathrm{e}-14$ & - & $1.50 \mathrm{e}-12$ & 6.03 & $6.21 \mathrm{e}-14$ & - & $1.56 \mathrm{e}-12$ & 5.99 & $1.56 \mathrm{e}-12$ & 5.99 \\
$\pi / 2^{7}$ & $1.54 \mathrm{e}-13$ & - & $3.98 \mathrm{e}-14$ & - & $1.54 \mathrm{e}-13$ & - & $1.02 \mathrm{e}-13$ & - & $5.40 \mathrm{e}-14$ & - & $1.02 \mathrm{e}-13$ & -
\end{tabular}

Table 7 Results for $\tau=\frac{i}{k h}$ and $\tau=\frac{1}{k h}$ for the SFH with $p=2$.

\begin{tabular}{|c|c|c|c|c|c|c|c|c|c|c|c|c|}
\hline \multirow[b]{2}{*}{$k h$} & \multicolumn{6}{|c|}{$\tau=\frac{i}{k h}$} & \multicolumn{6}{|c|}{$\tau=\frac{1}{k h}$} \\
\hline & $\epsilon_{\mathrm{disp}}$ & rate & $\epsilon_{\text {dissip }}$ & rate & $\epsilon_{\text {total }}$ & rate & $\epsilon_{\text {disp }}$ & rate & $\epsilon_{\text {dissip }}$ & rate & $\epsilon_{\text {total }}$ & rate \\
\hline$\pi / 2^{2}$ & $1.88 \mathrm{e}-05$ & - & $1.16 \mathrm{e}-15$ & - & $1.88 \mathrm{e}-05$ & - & $1.77 \mathrm{e}-06$ & - & $1.98 \mathrm{e}-05$ & - & $1.99 \mathrm{e}-05$ & - \\
\hline$\pi / 2^{3}$ & $1.58 \mathrm{e}-07$ & 6.90 & $1.90 \mathrm{e}-15$ & - & $1.58 \mathrm{e}-07$ & 6.90 & $5.35 \mathrm{e}-09$ & 8.37 & $1.58 \mathrm{e}-07$ & 6.97 & $1.58 \mathrm{e}-07$ & 6.97 \\
\hline$\pi / 2^{4}$ & $1.26 \mathrm{e}-09$ & 6.97 & $2.80 \mathrm{e}-15$ & - & $1.26 \mathrm{e}-09$ & 6.97 & $2.50 \mathrm{e}-11$ & 7.74 & $1.24 \mathrm{e}-09$ & 6.99 & $1.24 \mathrm{e}-09$ & 6.99 \\
\hline$\pi / 2^{5}$ & $9.87 \mathrm{e}-12$ & 6.99 & $1.30 \mathrm{e}-14$ & - & $9.87 \mathrm{e}-12$ & 6.99 & $1.64 \mathrm{e}-13$ & - & $9.71 \mathrm{e}-12$ & 7.00 & $9.71 \mathrm{e}-12$ & 7.00 \\
\hline$\pi / 2^{6}$ & $1.16 \mathrm{e}-13$ & - & $1.92 \mathrm{e}-14$ & - & $1.16 \mathrm{e}-13$ & - & $8.44 \mathrm{e}-14$ & - & $8.54 \mathrm{e}-14$ & - & $1.18 \mathrm{e}-13$ & - \\
\hline$\pi / 2^{7}$ & $8.72 \mathrm{e}-14$ & - & $1.79 \mathrm{e}-14$ & - & $8.90 \mathrm{e}-14$ & - & $1.40 \mathrm{e}-13$ & - & $3.30 \mathrm{e}-14$ & - & $1.44 \mathrm{e}-13$ & - \\
\hline
\end{tabular}

Table 8 Results for $\tau=i$ and $\tau=1$ for the SFH with $p=3$.

\begin{tabular}{l||cc|cc|cc||cc|cc|cc}
\multicolumn{1}{c||}{} & \multicolumn{9}{c||}{$\tau=i$} \\
$k h$ & $\epsilon_{\text {disp }}$ & rate & $\epsilon_{\text {dissip }}$ & rate & $\epsilon_{\text {total }}$ & rate & $\epsilon_{\text {disp }}$ & rate & $\epsilon_{\text {dissip }}$ & rate & $\epsilon_{\text {total }}$ & rate \\
\hline$\pi / 2^{2}$ & $1.20 \mathrm{e}-07$ & - & $2.70 \mathrm{e}-15$ & - & $1.20 \mathrm{e}-07$ & - & $1.12 \mathrm{e}-08$ & - & $1.25 \mathrm{e}-07$ & - & $1.26 \mathrm{e}-07$ & - \\
$\pi / 2^{3}$ & $4.87 \mathrm{e}-10$ & 7.94 & $2.80 \mathrm{e}-15$ & - & $4.87 \mathrm{e}-10$ & 7.94 & $2.23 \mathrm{e}-11$ & 8.98 & $4.99 \mathrm{e}-10$ & 7.97 & $4.99 \mathrm{e}-10$ & 7.98 \\
$\pi / 2^{4}$ & $1.94 \mathrm{e}-12$ & 7.97 & $4.62 \mathrm{e}-15$ & - & $1.94 \mathrm{e}-12$ & 7.97 & $3.29 \mathrm{e}-14$ & - & $1.96 \mathrm{e}-12$ & 7.99 & $1.96 \mathrm{e}-12$ & 7.99 \\
$\pi / 2^{5}$ & $6.06 \mathrm{e}-14$ & - & $1.35 \mathrm{e}-14$ & - & $6.06 \mathrm{e}-14$ & - & $1.03 \mathrm{e}-13$ & - & $1.16 \mathrm{e}-14$ & - & $1.03 \mathrm{e}-13$ & -
\end{tabular}

Table 9 Results for $\tau=\frac{i}{k h}$ and $\tau=\frac{1}{k h}$ for the SFH with $p=3$.

\begin{tabular}{l||cc|cc|cc||cc|cc|cc}
\multicolumn{1}{||}{} & \multicolumn{1}{c||}{$\tau=\frac{i}{k h}$} \\
$k h$ & $\epsilon_{\text {disp }}$ & rate & $\epsilon_{\text {dissip }}$ & rate & $\epsilon_{\text {total }}$ & rate & $\epsilon_{\text {disp }}$ & rate & $\epsilon_{\text {dissip }}$ & rate & $\epsilon_{\text {total }}$ & rate \\
\hline$\pi / 2^{2}$ & $9.61 \mathrm{e}-08$ & - & $9.47 e-16$ & - & $9.61 e-08$ & - & $7.89 \mathrm{e}-09$ & - & $9.84 \mathrm{e}-08$ & - & $9.88 \mathrm{e}-08$ & - \\
$\pi / 2^{3}$ & $1.98 \mathrm{e}-10$ & 8.92 & $2.71 e-15$ & - & $1.98 e-10$ & 8.92 & $7.60 \mathrm{e}-12$ & 10.02 & $1.96 \mathrm{e}-10$ & 8.97 & $1.96 \mathrm{e}-10$ & 8.98 \\
$\pi / 2^{4}$ & $3.95 \mathrm{e}-13$ & 8.97 & $3.44 e-15$ & - & $3.95 e-13$ & 8.97 & $5.99 \mathrm{e}-14$ & - & $3.81 \mathrm{e}-13$ & 9.01 & $3.85 \mathrm{e}-13$ & 8.99 \\
$\pi / 2^{5}$ & $6.25 \mathrm{e}-14$ & - & $5.77 e-15$ & - & $6.26 e-14$ & - & $2.50 \mathrm{e}-14$ & - & $8.20 \mathrm{e}-15$ & - & $2.52 \mathrm{e}-14$ & -
\end{tabular}

was already done in [12] for $p=0,1$ and $\tau=1, \sqrt{3} i / 2$. A natural question that arises, in view of the previously described results on $\mathrm{SFH}$ method, is whether the LDG-H exhibits better rates if the new-found parameter $\tau=i / k h$ is used.

To answer this, we compute the wavenumber errors of the LDG-H method for the same four values of $\tau$ we have been considering in Section 3-4 for $p=$ $0,1,2$ and 3 . The results are in Tables $10-17$. We find that for the imaginary values of $\tau$, the dissipative errors are zero. However, the best rates for the total error that we could observe was $O(h p)^{2 p+2}$, one order less than the best rates observed for the SFH method. We also note that the values of $\tau=i / k h$ and $\tau=1 / k h$ that gave better rates for the SFH method, do not give good rates for the LDG-H method. 
Table 10 Results for $\tau=i$ and $\tau=1$ for the LDG-H with $p=0$.

\begin{tabular}{l||cc|cc|cc||cc|cc|cc}
\multicolumn{1}{l||}{} & \multicolumn{9}{c||}{$\tau=i$} \\
$k h$ & $\epsilon_{\text {disp }}$ & rate & $\epsilon_{\text {dissip }}$ & rate & $\epsilon_{\text {total }}$ & rate & $\epsilon_{\text {disp }}$ & rate & $\epsilon_{\text {dissip }}$ & rate & $\epsilon_{\text {total }}$ & rate \\
\hline$\pi / 2^{2}$ & $1.41 \mathrm{e}-01$ & - & $6.39 \mathrm{e}-16$ & - & $1.41 \mathrm{e}-01$ & - & $6.16 \mathrm{e}-02$ & - & $1.74 \mathrm{e}-01$ & - & $1.84 \mathrm{e}-01$ & - \\
$\pi / 2^{3}$ & $3.08 \mathrm{e}-02$ & 2.20 & $1.77 \mathrm{e}-15$ & - & $3.08 \mathrm{e}-02$ & 2.20 & $8.73 \mathrm{e}-03$ & 2.82 & $4.80 \mathrm{e}-02$ & 1.85 & $4.88 \mathrm{e}-02$ & 1.92 \\
$\pi / 2^{4}$ & $7.23 \mathrm{e}-03$ & 2.09 & $1.44 \mathrm{e}-15$ & - & $7.23 \mathrm{e}-03$ & 2.09 & $1.13 \mathrm{e}-03$ & 2.95 & $1.23 \mathrm{e}-02$ & 1.96 & $1.24 \mathrm{e}-02$ & 1.98 \\
$\pi / 2^{5}$ & $1.75 \mathrm{e}-03$ & 2.04 & $1.55 \mathrm{e}-15$ & - & $1.75 \mathrm{e}-03$ & 2.04 & $1.42 \mathrm{e}-04$ & 2.99 & $3.11 \mathrm{e}-03$ & 1.99 & $3.11 \mathrm{e}-03$ & 1.99 \\
$\pi / 2^{6}$ & $4.32 \mathrm{e}-04$ & 2.02 & $3.44 \mathrm{e}-15$ & - & $4.32 \mathrm{e}-04$ & 2.02 & $1.79 \mathrm{e}-05$ & 3.00 & $7.78 \mathrm{e}-04$ & 2.00 & $7.79 \mathrm{e}-04$ & 2.00 \\
$\pi / 2^{7}$ & $1.07 \mathrm{e}-04$ & 2.01 & $1.60 \mathrm{e}-14$ & - & $1.07 \mathrm{e}-04$ & 2.01 & $2.23 \mathrm{e}-06$ & 3.00 & $1.95 \mathrm{e}-04$ & 2.00 & $1.95 \mathrm{e}-04$ & 2.00 \\
$\pi / 2^{8}$ & $2.67 \mathrm{e}-05$ & 2.01 & $2.29 \mathrm{e}-14$ & - & $2.67 \mathrm{e}-05$ & 2.01 & $2.79 \mathrm{e}-07$ & 3.00 & $4.87 \mathrm{e}-05$ & 2.00 & $4.87 \mathrm{e}-05$ & 2.00 \\
$\pi / 2^{9}$ & $6.67 \mathrm{e}-06$ & 2.00 & $3.00 \mathrm{e}-14$ & - & $6.67 \mathrm{e}-06$ & 2.00 & $3.49 \mathrm{e}-08$ & 3.00 & $1.22 \mathrm{e}-05$ & 2.00 & $1.22 \mathrm{e}-05$ & 2.00 \\
$\pi / 2^{10}$ & $1.67 \mathrm{e}-06$ & 2.00 & $1.01 \mathrm{e}-13$ & - & $1.67 \mathrm{e}-06$ & 2.00 & $4.36 \mathrm{e}-09$ & 3.00 & $3.04 \mathrm{e}-06$ & 2.00 & $3.04 \mathrm{e}-06$ & 2.00
\end{tabular}

Table 11 Results for $\tau=\frac{i}{k h}$ and $\tau=\frac{1}{k h}$ for the LDG-H with $p=0$.

\begin{tabular}{l||cc|cc|cc||cc|cc|cc}
\multicolumn{1}{l||}{} & \multicolumn{9}{c||}{$\tau$} & \multicolumn{4}{c}{$\tau=\frac{1}{k h}$} \\
$k h$ & $\epsilon_{\text {disp }}$ & rate & $\epsilon_{\text {dissip }}$ & rate & $\epsilon_{\text {total }}$ & rate & $\epsilon_{\text {disp }}$ & rate & $\epsilon_{\text {dissip }}$ & rate & $\epsilon_{\text {total }}$ & rate \\
\hline$\pi / 2^{2}$ & $2.39 \mathrm{e}-01$ & - & $7.28 \mathrm{e}-16$ & - & $2.39 \mathrm{e}-01$ & - & $7.97 \mathrm{e}-02$ & - & $1.93 \mathrm{e}-01$ & - & $2.09 \mathrm{e}-01$ & - \\
$\pi / 2^{3}$ & $1.51 \mathrm{e}-01$ & 0.67 & $1.46 \mathrm{e}-15$ & - & $1.51 \mathrm{e}-01$ & 0.67 & $3.33 \mathrm{e}-02$ & 1.26 & $8.83 \mathrm{e}-02$ & 1.13 & $9.43 \mathrm{e}-02$ & 1.15 \\
$\pi / 2^{4}$ & $7.98 \mathrm{e}-02$ & 0.92 & $1.37 \mathrm{e}-15$ & - & $7.98 \mathrm{e}-02$ & 0.92 & $1.59 \mathrm{e}-02$ & 1.07 & $4.30 \mathrm{e}-02$ & 1.04 & $4.59 \mathrm{e}-02$ & 1.04 \\
$\pi / 2^{5}$ & $4.05 \mathrm{e}-02$ & 0.98 & $3.07 \mathrm{e}-15$ & - & $4.05 \mathrm{e}-02$ & 0.98 & $7.84 \mathrm{e}-03$ & 1.02 & $2.14 \mathrm{e}-02$ & 1.01 & $2.28 \mathrm{e}-02$ & 1.01 \\
$\pi / 2^{6}$ & $2.03 \mathrm{e}-02$ & 0.99 & $7.53 \mathrm{e}-15$ & - & $2.03 \mathrm{e}-02$ & 0.99 & $3.91 \mathrm{e}-03$ & 1.00 & $1.07 \mathrm{e}-02$ & 1.00 & $1.14 \mathrm{e}-02$ & 1.00 \\
$\pi / 2^{7}$ & $1.02 \mathrm{e}-02$ & 1.00 & $1.09 \mathrm{e}-14$ & - & $1.02 \mathrm{e}-02$ & 1.00 & $1.95 \mathrm{e}-03$ & 1.00 & $5.33 \mathrm{e}-03$ & 1.00 & $5.68 \mathrm{e}-03$ & 1.00 \\
$\pi / 2^{8}$ & $5.08 \mathrm{e}-03$ & 1.00 & $2.73 \mathrm{e}-14$ & - & $5.08 \mathrm{e}-03$ & 1.00 & $9.76 \mathrm{e}-04$ & 1.00 & $2.67 \mathrm{e}-03$ & 1.00 & $2.84 \mathrm{e}-03$ & 1.00 \\
$\pi / 2^{9}$ & $2.54 \mathrm{e}-03$ & 1.00 & $6.68 \mathrm{e}-14$ & - & $2.54 \mathrm{e}-03$ & 1.00 & $4.88 \mathrm{e}-04$ & 1.00 & $1.33 \mathrm{e}-03$ & 1.00 & $1.42 \mathrm{e}-03$ & 1.00 \\
$\pi / 2^{10}$ & $1.27 \mathrm{e}-03$ & 1.00 & $6.10 \mathrm{e}-14$ & - & $1.27 \mathrm{e}-03$ & 1.00 & $2.44 \mathrm{e}-04$ & 1.00 & $6.67 \mathrm{e}-04$ & 1.00 & $7.10 \mathrm{e}-04$ & 1.00
\end{tabular}

Table 12 Results for $\tau=i$ and $\tau=1$ for the LDG-H with $p=1$.

\begin{tabular}{l||cc|cc|cc||cc|cc|cc}
\multicolumn{1}{l||}{} & \multicolumn{9}{c||}{$\tau=i$} \\
$k h$ & $\epsilon_{\text {disp }}$ & rate & $\epsilon_{\text {dissip }}$ & rate & $\epsilon_{\text {total }}$ & rate & $\epsilon_{\text {disp }}$ & rate & $\epsilon_{\text {dissip }}$ & rate & $\epsilon_{\text {total }}$ & rate \\
\hline$\pi / 2^{2}$ & $2.90 \mathrm{e}-03$ & - & $4.80 \mathrm{e}-16$ & - & $2.90 \mathrm{e}-03$ & - & $7.69 \mathrm{e}-04$ & - & $4.82 \mathrm{e}-03$ & - & $4.88 \mathrm{e}-03$ & - \\
$\pi / 2^{3}$ & $1.79 \mathrm{e}-04$ & 4.02 & $1.40 \mathrm{e}-15$ & - & $1.79 \mathrm{e}-04$ & 4.02 & $2.37 \mathrm{e}-05$ & 5.02 & $3.16 \mathrm{e}-04$ & 3.93 & $3.17 \mathrm{e}-04$ & 3.95 \\
$\pi / 2^{4}$ & $1.11 \mathrm{e}-05$ & 4.01 & $3.01 \mathrm{e}-15$ & - & $1.11 \mathrm{e}-05$ & 4.01 & $7.36 \mathrm{e}-07$ & 5.01 & $1.99 \mathrm{e}-05$ & 3.98 & $2.00 \mathrm{e}-05$ & 3.99 \\
$\pi / 2^{5}$ & $6.89 \mathrm{e}-07$ & 4.01 & $4.10 \mathrm{e}-15$ & - & $6.89 \mathrm{e}-07$ & 4.01 & $2.30 \mathrm{e}-08$ & 5.00 & $1.25 \mathrm{e}-06$ & 4.00 & $1.25 \mathrm{e}-06$ & 4.00 \\
$\pi / 2^{6}$ & $4.29 \mathrm{e}-08$ & 4.00 & $4.50 \mathrm{e}-15$ & - & $4.29 \mathrm{e}-08$ & 4.00 & $7.18 \mathrm{e}-10$ & 5.00 & $7.82 \mathrm{e}-08$ & 4.00 & $7.82 \mathrm{e}-08$ & 4.00 \\
$\pi / 2^{7}$ & $2.68 \mathrm{e}-09$ & 4.00 & $4.00 \mathrm{e}-14$ & - & $2.68 \mathrm{e}-09$ & 4.00 & $2.24 \mathrm{e}-11$ & 5.00 & $4.89 \mathrm{e}-09$ & 4.00 & $4.89 \mathrm{e}-09$ & 4.00 \\
$\pi / 2^{8}$ & $1.67 \mathrm{e}-10$ & 4.00 & $3.54 \mathrm{e}-14$ & - & $1.67 \mathrm{e}-10$ & 4.00 & $9.13 \mathrm{e}-13$ & 4.62 & $3.05 \mathrm{e}-10$ & 4.00 & $3.05 \mathrm{e}-10$ & 4.00 \\
$\pi / 2^{9}$ & $1.04 \mathrm{e}-11$ & 4.01 & $5.14 \mathrm{e}-14$ & - & $1.04 \mathrm{e}-11$ & 4.01 & $6.05 \mathrm{e}-13$ & - & $1.91 \mathrm{e}-11$ & 4.00 & $1.91 \mathrm{e}-11$ & 4.00 \\
$\pi / 2^{10}$ & $1.47 \mathrm{e}-12$ & - & $1.14 \mathrm{e}-13$ & - & $1.47 \mathrm{e}-12$ & - & $9.16 \mathrm{e}-13$ & - & $1.20 \mathrm{e}-12$ & 3.99 & $1.49 \mathrm{e}-12$ & 3.68
\end{tabular}

All the rates observed individually in Tables $10-17$ are summarized together in Table 1 of the introduction, where the cases from other sections are also included to facilitate comparison. 
Table 13 Results for $\tau=\frac{i}{k h}$ and $\tau=\frac{1}{k h}$ for the LDG-H with $p=1$.

\begin{tabular}{l||cc|cc|cc||cc|cc|cc}
\multicolumn{1}{l||}{} & \multicolumn{9}{c||}{$\tau=\frac{}{k}$} \\
$k h$ & $\epsilon_{\text {disp }}$ & rate & $\epsilon_{\text {dissip }}$ & rate & $\epsilon_{\text {total }}$ & rate & $\epsilon_{\text {disp }}$ & rate & $\epsilon_{\text {dissip }}$ & rate & $\epsilon_{\text {total }}$ & rate \\
\hline$\pi / 2^{2}$ & $4.47 \mathrm{e}-03$ & - & $4.63 \mathrm{e}-16$ & - & $4.47 \mathrm{e}-03$ & - & $9.86 \mathrm{e}-04$ & - & $5.57 \mathrm{e}-03$ & - & $5.65 \mathrm{e}-03$ & - \\
$\pi / 2^{3}$ & $6.80 \mathrm{e}-04$ & 2.71 & $1.59 \mathrm{e}-15$ & - & $6.80 \mathrm{e}-04$ & 2.71 & $8.79 \mathrm{e}-05$ & 3.49 & $6.40 \mathrm{e}-04$ & 3.12 & $6.46 \mathrm{e}-04$ & 3.13 \\
$\pi / 2^{4}$ & $8.90 \mathrm{e}-05$ & 2.93 & $1.36 \mathrm{e}-15$ & - & $8.90 \mathrm{e}-05$ & 2.93 & $9.98 \mathrm{e}-06$ & 3.14 & $7.81 \mathrm{e}-05$ & 3.03 & $7.88 \mathrm{e}-05$ & 3.04 \\
$\pi / 2^{5}$ & $1.13 \mathrm{e}-05$ & 2.98 & $5.10 \mathrm{e}-15$ & - & $1.13 \mathrm{e}-05$ & 2.98 & $1.22 \mathrm{e}-06$ & 3.04 & $9.71 \mathrm{e}-06$ & 3.01 & $9.78 \mathrm{e}-06$ & 3.01 \\
$\pi / 2^{6}$ & $1.41 \mathrm{e}-06$ & 3.00 & $6.11 \mathrm{e}-15$ & - & $1.41 \mathrm{e}-06$ & 3.00 & $1.51 \mathrm{e}-07$ & 3.01 & $1.21 \mathrm{e}-06$ & 3.00 & $1.22 \mathrm{e}-06$ & 3.00 \\
$\pi / 2^{7}$ & $1.76 \mathrm{e}-07$ & 3.00 & $1.98 \mathrm{e}-14$ & - & $1.76 \mathrm{e}-07$ & 3.00 & $1.89 \mathrm{e}-08$ & 3.00 & $1.51 \mathrm{e}-07$ & 3.00 & $1.53 \mathrm{e}-07$ & 3.00 \\
$\pi / 2^{8}$ & $2.21 \mathrm{e}-08$ & 3.00 & $5.99 \mathrm{e}-14$ & - & $2.21 \mathrm{e}-08$ & 3.00 & $2.36 \mathrm{e}-09$ & 3.00 & $1.89 \mathrm{e}-08$ & 3.00 & $1.91 \mathrm{e}-08$ & 3.00 \\
$\pi / 2^{9}$ & $2.76 \mathrm{e}-09$ & 3.00 & $5.11 \mathrm{e}-14$ & - & $2.76 \mathrm{e}-09$ & 3.00 & $2.94 \mathrm{e}-10$ & 3.00 & $2.36 \mathrm{e}-09$ & 3.00 & $2.38 \mathrm{e}-09$ & 3.00 \\
$\pi / 2^{10}$ & $3.45 \mathrm{e}-10$ & 3.00 & $1.77 \mathrm{e}-13$ & - & $3.45 \mathrm{e}-10$ & 3.00 & $3.74 \mathrm{e}-11$ & 2.98 & $2.96 \mathrm{e}-10$ & 3.00 & $2.98 \mathrm{e}-10$ & 3.00
\end{tabular}

Table 14 Results for $\tau=i$ and $\tau=1$ for the LDG-H with $p=2$.

\begin{tabular}{l||cc|cc|cc||cc|cc|cc}
\multicolumn{1}{l||}{} & \multicolumn{9}{c||}{$\tau=i$} \\
$k h$ & $\epsilon_{\text {disp }}$ & rate & $\epsilon_{\text {dissip }}$ & rate & $\epsilon_{\text {total }}$ & rate & $\epsilon_{\text {disp }}$ & rate & $\epsilon_{\text {dissip }}$ & rate & $\epsilon_{\text {total }}$ & rate \\
\hline$\pi / 2^{2}$ & $2.64 \mathrm{e}-05$ & - & $8.49 \mathrm{e}-16$ & - & $2.64 \mathrm{e}-05$ & - & $5.58 \mathrm{e}-06$ & - & $4.54 \mathrm{e}-05$ & - & $4.58 \mathrm{e}-05$ & - \\
$\pi / 2^{3}$ & $4.12 \mathrm{e}-07$ & 6.01 & $2.82 \mathrm{e}-15$ & - & $4.12 \mathrm{e}-07$ & 6.01 & $4.45 \mathrm{e}-08$ & 6.97 & $7.33 \mathrm{e}-07$ & 5.95 & $7.34 \mathrm{e}-07$ & 5.96 \\
$\pi / 2^{4}$ & $6.39 \mathrm{e}-09$ & 6.01 & $7.56 \mathrm{e}-15$ & - & $6.39 \mathrm{e}-09$ & 6.01 & $3.50 \mathrm{e}-10$ & 6.99 & $1.15 \mathrm{e}-08$ & 5.99 & $1.16 \mathrm{e}-08$ & 5.99 \\
$\pi / 2^{5}$ & $9.94 \mathrm{e}-11$ & 6.01 & $7.11 \mathrm{e}-15$ & - & $9.94 \mathrm{e}-11$ & 6.01 & $2.72 \mathrm{e}-12$ & 7.01 & $1.81 \mathrm{e}-10$ & 6.00 & $1.81 \mathrm{e}-10$ & 6.00 \\
$\pi / 2^{6}$ & $1.63 \mathrm{e}-12$ & 5.93 & $5.96 \mathrm{e}-15$ & - & $1.63 \mathrm{e}-12$ & 5.93 & $3.44 \mathrm{e}-14$ & 6.30 & $2.83 \mathrm{e}-12$ & 6.00 & $2.83 \mathrm{e}-12$ & 6.00 \\
$\pi / 2^{7}$ & $1.22 \mathrm{e}-13$ & - & $7.05 \mathrm{e}-14$ & - & $1.22 \mathrm{e}-13$ & - & $1.28 \mathrm{e}-13$ & - & $6.86 \mathrm{e}-14$ & - & $1.36 \mathrm{e}-13$ & -
\end{tabular}

Table 15 Results for $\tau=\frac{i}{k h}$ and $\tau=\frac{1}{k h}$ for the LDG-H with $p=2$.

\begin{tabular}{|c|c|c|c|c|c|c|c|c|c|c|c|c|}
\hline \multirow[b]{2}{*}{$k h$} & \multicolumn{6}{|c|}{$\tau=\frac{i}{k h}$} & \multicolumn{6}{|c|}{$\tau=\frac{1}{k h}$} \\
\hline & $\epsilon_{\text {disp }}$ & rate & $\epsilon_{\text {dissip }}$ & rate & $\epsilon_{\text {total }}$ & rate & $\epsilon_{\text {disp }}$ & rate & $\epsilon_{\text {dissip }}$ & rate & $\epsilon_{\text {total }}$ & rate \\
\hline$\pi / 2^{2}$ & $4.04 e-05$ & - & $1.34 e-15$ & - & $4.04 e-05$ & - & $7.23 \mathrm{e}-06$ & - & $5.26 \mathrm{e}-05$ & - & $5.31 \mathrm{e}-05$ & - \\
\hline$\pi / 2^{3}$ & $1.54 e-06$ & 4.72 & $1.40 e-15$ & - & $1.54 e-06$ & 4.72 & $1.68 \mathrm{e}-07$ & 5.42 & $1.49 \mathrm{e}-06$ & 5.14 & $1.50 \mathrm{e}-06$ & 5.14 \\
\hline$\pi / 2^{4}$ & $5.03 e-08$ & 4.93 & $3.88 e-15$ & - & $5.03 e-08$ & 4.93 & $4.85 \mathrm{e}-09$ & 5.12 & $4.54 \mathrm{e}-08$ & 5.04 & $4.57 \mathrm{e}-08$ & 5.04 \\
\hline$\pi / 2^{5}$ & $1.59 e-09$ & 4.98 & $1.35 e-14$ & - & $1.59 e-09$ & 4.98 & $1.48 \mathrm{e}-10$ & 5.03 & $1.41 \mathrm{e}-09$ & 5.01 & $1.42 \mathrm{e}-09$ & 5.01 \\
\hline$\pi / 2^{6}$ & $4.98 e-11$ & 5.00 & $1.27 e-14$ & - & $4.98 e-11$ & 5.00 & $4.61 \mathrm{e}-12$ & 5.01 & $4.40 \mathrm{e}-11$ & 5.00 & $4.43 \mathrm{e}-11$ & 5.00 \\
\hline$\pi / 2^{7}$ & $1.80 e-12$ & 4.79 & $3.33 e-14$ & - & $1.80 e-12$ & 4.79 & $1.63 \mathrm{e}-13$ & 4.82 & $1.41 \mathrm{e}-12$ & 4.96 & $1.41 \mathrm{e}-12$ & 4.97 \\
\hline
\end{tabular}

Table 16 Results for $\tau=i$ and $\tau=1$ for the LDG-H with $p=3$.

\begin{tabular}{l||cc|cc|cc||cc|cc|cc}
\multicolumn{1}{c||}{} & \multicolumn{9}{c||}{$\tau=i$} \\
$k h$ & $\epsilon_{\text {disp }}$ & rate & $\epsilon_{\text {dissip }}$ & rate & $\epsilon_{\text {total }}$ & rate & $\epsilon_{\text {disp }}$ & rate & $\epsilon_{\text {dissip }}$ & rate & $\epsilon_{\text {total }}$ & rate \\
\hline$\pi / 2^{2}$ & $1.30 \mathrm{e}-07$ & - & $1.54 \mathrm{e}-15$ & - & $1.30 \mathrm{e}-07$ & - & $2.34 \mathrm{e}-08$ & - & $2.27 \mathrm{e}-07$ & - & $2.28 \mathrm{e}-07$ & - \\
$\pi / 2^{3}$ & $5.08 \mathrm{e}-10$ & 8.00 & $1.87 \mathrm{e}-15$ & - & $5.08 \mathrm{e}-10$ & 8.00 & $4.68 \mathrm{e}-11$ & 8.97 & $9.10 \mathrm{e}-10$ & 7.96 & $9.11 \mathrm{e}-10$ & 7.97 \\
$\pi / 2^{4}$ & $2.00 \mathrm{e}-12$ & 7.99 & $5.43 \mathrm{e}-15$ & - & $2.00 \mathrm{e}-12$ & 7.99 & $1.14 \mathrm{e}-13$ & 8.68 & $3.58 \mathrm{e}-12$ & 7.99 & $3.58 \mathrm{e}-12$ & 7.99 \\
$\pi / 2^{5}$ & $7.71 \mathrm{e}-14$ & - & $9.44 \mathrm{e}-15$ & - & $7.75 \mathrm{e}-14$ & - & $5.68 \mathrm{e}-14$ & - & $1.91 \mathrm{e}-14$ & - & $5.99 \mathrm{e}-14$ & -
\end{tabular}

Table 17 Results for $\tau=\frac{i}{k h}$ and $\tau=\frac{1}{k h}$ for the LDG-H with $p=3$.

\begin{tabular}{l||cc|cc|cc||cc|cc|cc}
\multicolumn{1}{c||}{} & \multicolumn{9}{c||}{$\tau=\frac{}{k}$} & \multicolumn{5}{c}{$\tau=\frac{1}{k h}$} \\
$k h$ & $\epsilon_{\text {disp }}$ & rate & $\epsilon_{\text {dissip }}$ & rate & $\epsilon_{\text {total }}$ & rate & $\epsilon_{\text {disp }}$ & rate & $\epsilon_{\text {dissip }}$ & rate & $\epsilon_{\text {total }}$ & rate \\
\hline$\pi / 2^{2}$ & $1.98 \mathrm{e}-07$ & - & $8.52 \mathrm{e}-16$ & - & $1.98 \mathrm{e}-07$ & - & $3.04 \mathrm{e}-08$ & - & $2.63 \mathrm{e}-07$ & - & $2.65 \mathrm{e}-07$ & - \\
$\pi / 2^{3}$ & $1.87 \mathrm{e}-09$ & 6.72 & $2.35 \mathrm{e}-15$ & - & $1.87 \mathrm{e}-09$ & 6.72 & $1.78 \mathrm{e}-10$ & 7.41 & $1.86 \mathrm{e}-09$ & 7.15 & $1.87 \mathrm{e}-09$ & 7.15 \\
$\pi / 2^{4}$ & $1.53 \mathrm{e}-11$ & 6.94 & $9.38 \mathrm{e}-15$ & - & $1.53 \mathrm{e}-11$ & 6.94 & $1.28 \mathrm{e}-12$ & 7.12 & $1.41 \mathrm{e}-11$ & 7.04 & $1.42 \mathrm{e}-11$ & 7.04 \\
$\pi / 2^{5}$ & $6.20 \mathrm{e}-14$ & - & $1.05 \mathrm{e}-14$ & - & $6.20 \mathrm{e}-14$ & - & $7.77 \mathrm{e}-14$ & - & $1.16 \mathrm{e}-13$ & - & $1.27 \mathrm{e}-13$ & -
\end{tabular}


Acknowledgements This paper is the outgrowth of a suggestion from Prof. Bernardo Cockburn to go beyond the standard HDG method while comparing dispersion relations. On this occasion for celebrating Prof. Cockburn's contributions, the authors would like to place on record their deep appreciation for his tireless efforts to nurture the mathematical community of researchers in discontinuous Galerkin methods over the years.

This work was initiated while the student author F. Vargas was visiting Portland State University, thanks to the support from CONICYT, Chile. M. Solano was partially supported by Conicyt-Chile through Fondecyt project No. 1160320, BASAL project CMM and $\mathrm{CI}^{2} \mathrm{MA}$.

\section{References}

1. Ainsworth, M.: Discrete dispersion relation for $h p$-version finite element approximation at high wave number. SIAM Journal on Numerical Analysis 42(2), 553-575 (2004)

2. Ainsworth, M.: Dispersive and dissipative behaviour of high order discontinuous Galerkin finite element methods. J. Comput. Phys. 198(1), 106-130 (2004)

3. Ainsworth, M., Monk, P., Muniz, W.: Dispersive and dissipative properties of discontinuous Galerkin finite element methods for the second order wave equation. Journal of Scientific Computing 27(1-3), 5-40 (2006)

4. Babuška, I.M., Sauter, S.A.: Is the pollution effect of the FEM avoidable for the Helmholtz equation considering high wave numbers? SIAM Journal on Numerical Analysis 34(6), 2392-2423 (1997)

5. Cockburn, B., Dong, B., Guzmán, J.: A superconvergent LDG-hybridizable Galerkin method for second-order elliptic problems. Mathematics of Computation 77(264), 1887$1916(2008)$

6. Cockburn, B., Gopalakrishnan, J., Lazarov, R.: Unified hybridization of discontinuous Galerkin, mixed, and continuous Galerkin methods for second order elliptic problems. SIAM Journal on Numerical Analysis 47(2), 1319-1365 (2009)

7. Cui, J., Zhang, W.: An analysis of HDG methods for the Helmholtz equation. IMA Journal of Numerical Analysis 34(1), 279-295 (2014)

8. De Basabe, J.D., Sen, M.K., Wheeler, M.F.: The interior penalty discontinuous galerkin method for elastic wave propagation: grid dispersion. Geophysical Journal International 175(1), 83-93 (2008)

9. Deraemaeker, A., Babuška, I.M., Bouillard, P.: Dispersion and pollution of the FEM solution for the helmholtz equation in one, two and three dimensions. International Journal for Numerical Methods in Engineering 46(4), 471-499 (1999)

10. Giorgiani, G., Fernández-Méndez, S., Huerta, A.: Hybridizable discontinuous Galerkin p-adaptivity for wave propagation problems. International Journal for Numerical Methods in Fluids $\mathbf{7 2}(12), 1244-1262$ (2013)

11. Gittelson, C.J., Hiptmair, R.: Dispersion analysis of plane wave discontinuous Galerkin methods. International Journal for Numerical Methods in Engineering 98(5), 313-323 (2014)

12. Gopalakrishnan, J., Lanteri, S., Olivares, N., Perrussel, R.: Stabilization in relation to wavenumber in HDG methods. Advanced Modeling and Simulation in Engineering Sciences 2(1), 13 (2015)

13. Gopalakrishnan, J., Muga, I., Olivares, N.: Dispersive and dissipative errors in the DPG method with scaled norms for the Helmholtz equation. SIAM J. Sci. Comput. 36(1), A20-A39 (2014)

14. Griesmaier, R., Monk, P.: Error analysis for a hybridizable discontinuous Galerkin method for the Helmholtz equation. Journal of Scientific Computing 49(2), 291-310 (2011)

15. Hu, F.Q., Hussaini, M., Rasetarinera, P.: An analysis of the discontinuous Galerkin method for wave propagation problems. Journal of Computational Physics 151(2), 921-946 (1999)

16. Kirby, R.C.: Singularity-free evaluation of collapsed-coordinate orthogonal polynomials. ACM Trans. Math. Softw. 37(5) (2010)

17. Sherwin, S.: Dispersion Analysis of the Continuous and Discontinuous Galerkin Formulations, pp. 425-431. Springer Berlin Heidelberg, Berlin, Heidelberg (2000) 